\title{
Dosimetric evaluation of a 320 detector row CT scanner unit
}

\author{
Mario de Denaro and Paola Bregant \\ S.C. di Fisica Sanitaria, A.O.U. “Ospedali Riuniti” di Trieste, Trieste, Italy
}

Received 22 May 2010

Accepted 2 September 2010

Correspondence to: Dr. Mario de Denaro, Struttura Complessa di Fisica Sanitaria, Azienda Ospedaliero Universitaria “Ospedali Riuniti” di Trieste, Ospedale Maggiore, Centro Tumori, Via Pietà 19, 34100 Trieste, Italy. Phone: +39 040399 2341; Fax: +39 040 399 2367; E-mail: mario.dedenaro@aots.sanita.fvg.it

Disclosure: No potential conflicts of interest were disclosed.

Background. The technologic improvements in Multislice scanners include the increment in the X-ray beam width. Some new CT scanners are equipped with a 320 detector row which allows a longitudinal coverage of $160 \mathrm{~mm}$ and a total of 640 slices for a single rotation. When such parameters are used the length of the traditional pencil chamber ( 10

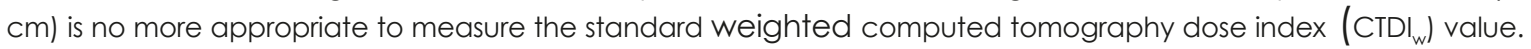
Materials and methods. Dosimetric measurements were performed on a 640 slices Toshiba Aquilion One CT scanner using common instrumentation available in Medical Physics Departments.

Results. For the measurements in air, two different ionization chambers were completely exposed to the beam. Dosimeters showed an acceptable agreement in the measurements. To evaluate the actual shape of the dose profile strips of Gafchromic XRQA film were used. Films were previously calibrated on site. From the graphic response of the scanned film it is possible to evaluate the full width at half maximum (FWHM) of the dose profile which represent the actual beam width.

Conclusions. Computed Tomography Dose Index (CTDI) and Dose Length Product (DLP) need to be changed when the beam width of the CT scanner is over $100 \mathrm{~mm}$. To perform dose evaluation with the conventional instrumentation, two parameters should be considered: the average absorbed dose and the actual beam width. To measure the average absorbed dose, the conventional ionization chamber can be used. For the measurement of the width of the dose profile, Gafchromic XRQA film seemed to be suitable.

Key words: multislice CT; pencil chamber; computed tomography dose index; dose profile; Gafchromic film

\section{Introduction}

The advent of multislice scanners technology ${ }^{1}$ leads to a continuous increment of the $\mathrm{X}$-ray beam width along the cranio-caudal direction. The new CT scanner from Toshiba, Aquilion One, is equipped with the 320 detector rows each one $0.5 \mathrm{~mm}$ wide. This new technology allows a longitudinal coverage of $160 \mathrm{~mm}$ at rotation time of 0.35 seconds. The important change in dosimetric evaluation is needed to adapt the CT dosimetry metrics to the new standard. ${ }^{2-5}$ With a $160 \mathrm{~mm}$ beam width as an operating condition, the length of the traditional pencil chamber $(10 \mathrm{~cm})$ is no more appropriate to evaluate the standard computed tomography dose index (CTDI). ${ }^{6}$ Despite this problem the CTDI and the dose length product (DLP) values are still dis- played on the scanner console. The problem for medical physicists, who must carry out quality assurance and dose optimization on the new CT equipment, is that suitable instrumentation is usually not available. In the present work we suggest some dosimetric measurements that can be carried out on a 320 row CT scanner unit, by means of conventional dosimetric instrumentation.

\section{Material and methods}

Some measurements on a 640 slices Toshiba Aquilion One CT scanner were carried out by means of common instrumentation typically employed at Medical Physics Departments. CT scanners providing $160 \mathrm{~mm}$ beam width allows to completely 
expose the 6cc ionization chamber of a Radcal Dosimeter, model 9015, commonly employed in the conventional radiodiagnostic measurement. To evaluate the average absorbed dose due to the primary beam, we compared the measurement carried out in air at the isocenter of the gantry, during a single rotation, exposing together the Radcal Dosimeter and the standard $10 \mathrm{~cm}$ long CT pencil chamber (WDCT 10, Wellhofer, Germany), connected to PMX-III electrometer. The average absorbed dose alone does not give information regarding the profile of the dose along the craniocaudal direction ( $\mathrm{Z}$ axis); it is a parameter to be considered to evaluate the shape and the actual width of the beam. To measure the dose profile free in air Gafchromic XRQA film was exposed to the primary beam during a single axial rotation. The film was previously calibrated exposing small pieces of Gafchromic $(2 \times 2 \mathrm{~cm})$ to increasing doses; the Gafchromic films were put close to a reference ionization chamber. A calibration curve specific for the CT scanner beam quality was then obtained and it was used to convert the pixel values of the film into dose values (Figure 1). ${ }^{7}$

The exposed film was then scanned by a regular scanner (Epson 1680 pro) using red-green-blue (RGB) modality at 48 bits of depth and spatial resolution of 72 dots per inch. Only the red component of the image was considered. The dose profile was obtained correcting the pixel values by the calibration curve (Figure 2).

From the graph the full width at half maximum (FWHM) of the dose profile can be evaluated. FWHM is a parameter which is related to the Z-axis geometric efficiency. CTDI ${ }_{\text {air }, 300}$ (CTDI value using an integration interval of $300 \mathrm{~mm}$ ) and CTDI ${ }_{\text {air, } 100}$ (CTDI value using an integration interval of 100 $\mathrm{mm}$ ) were evaluated and compared (Figure 3 ). ${ }^{2}$

Finally, we put a strip of Gafchromic film in the holes of a standard CT dosimetric PMMA phantom (Figure 4) to evaluate the average absorbed dose inside the phantom (Figure 5).

\section{Results}

The measurement performed by the Radcal dosimeter showed an acceptable agreement with the values obtained by the Wellhofer pencil chamber (Table 1). The comparison was meaningful because the 6 cc chamber of the Radcal dosimeter is completely exposed by the beam width.

To maintain the meaning of the CTDI, the integration interval for $160 \mathrm{~mm}$ beam width must be

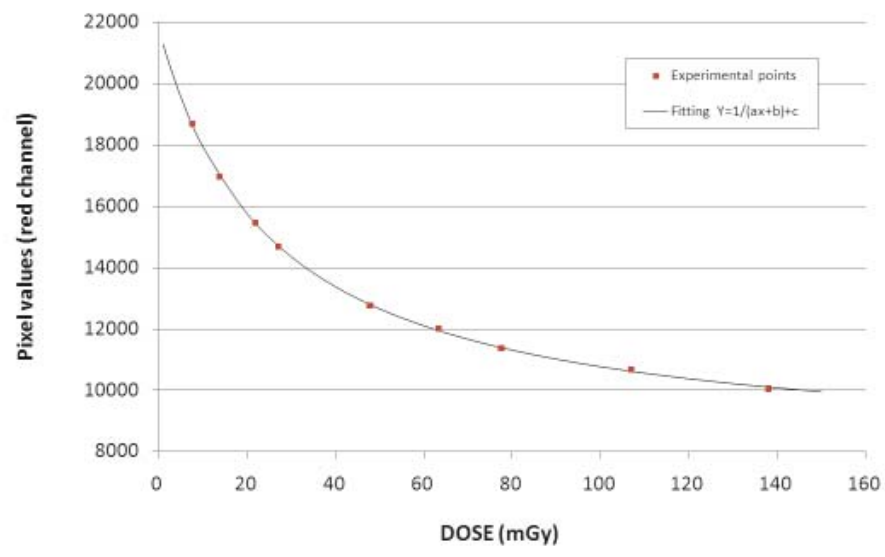

FIGURE 1. Fitting curve for the calibration of Gafchromic XRQA film.

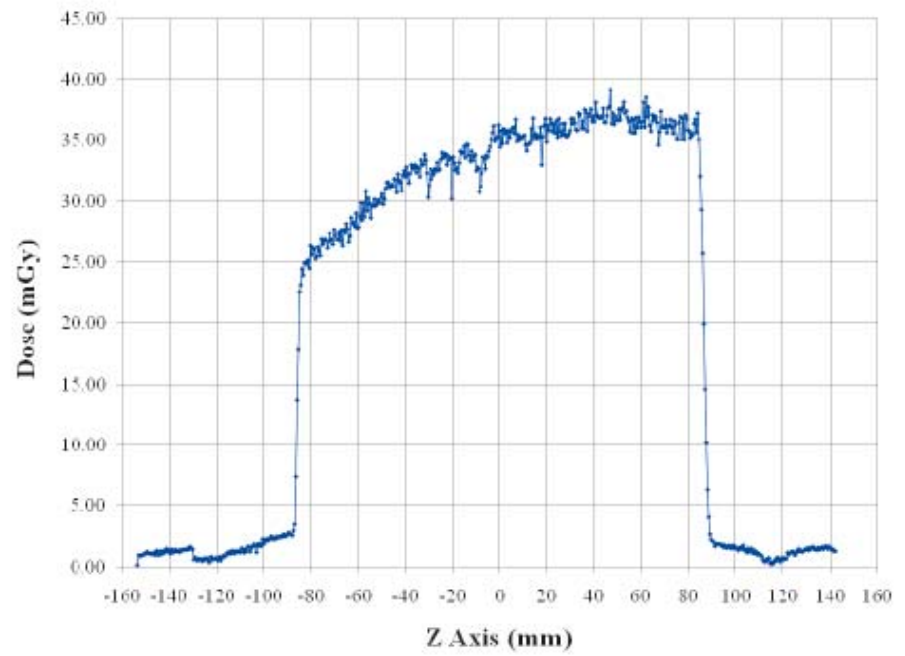

FIGURE 2. Dose profile measured by Gafchromic XRQA film.
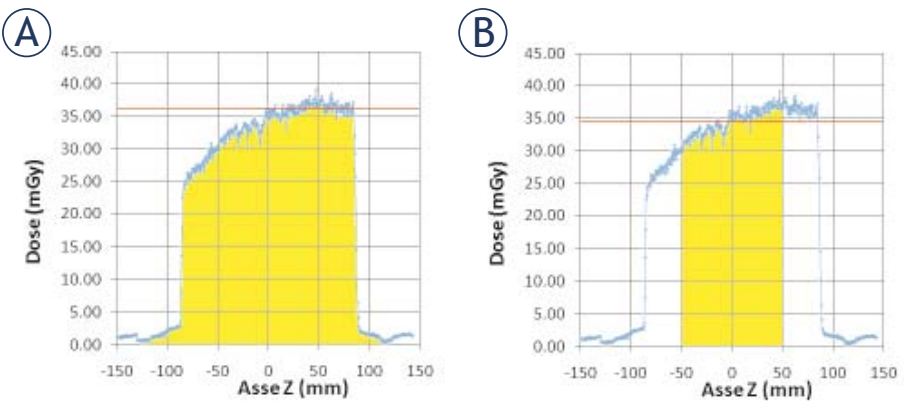

FIGURE 3. (A) Computed tomography dose index (CTDI)air,300. (B) CTDlair, 100.

extended to a value of at least $300 \mathrm{~mm} .^{2}$ For the measurement of the weighted computed tomography dose index $\left(\mathrm{CTDI}_{\mathrm{w}}\right)$ a PMMA phantom is required, but at the moment it is very difficult to find and manage a PMMA phantom with a length 
TABLE 1. Absorbed dose evaluated with two different dosimeters

\begin{tabular}{lcc}
\hline Dosimeter & Dose FOV Head (mGy/mAs) & Dose FOV Body (mGy/mAs) \\
\hline Wellhöfer pencil chamber (WDCT 10), with PMX-III & 0.257 & 0.225 \\
Radcal model 9015with 6cc chamber 10X5-6 & 0.272 & 0.243 \\
\hline
\end{tabular}

FOV, field-of-view; WDCT 10, Wellhöfer CT pencil chamber $10 \mathrm{~cm}$ long

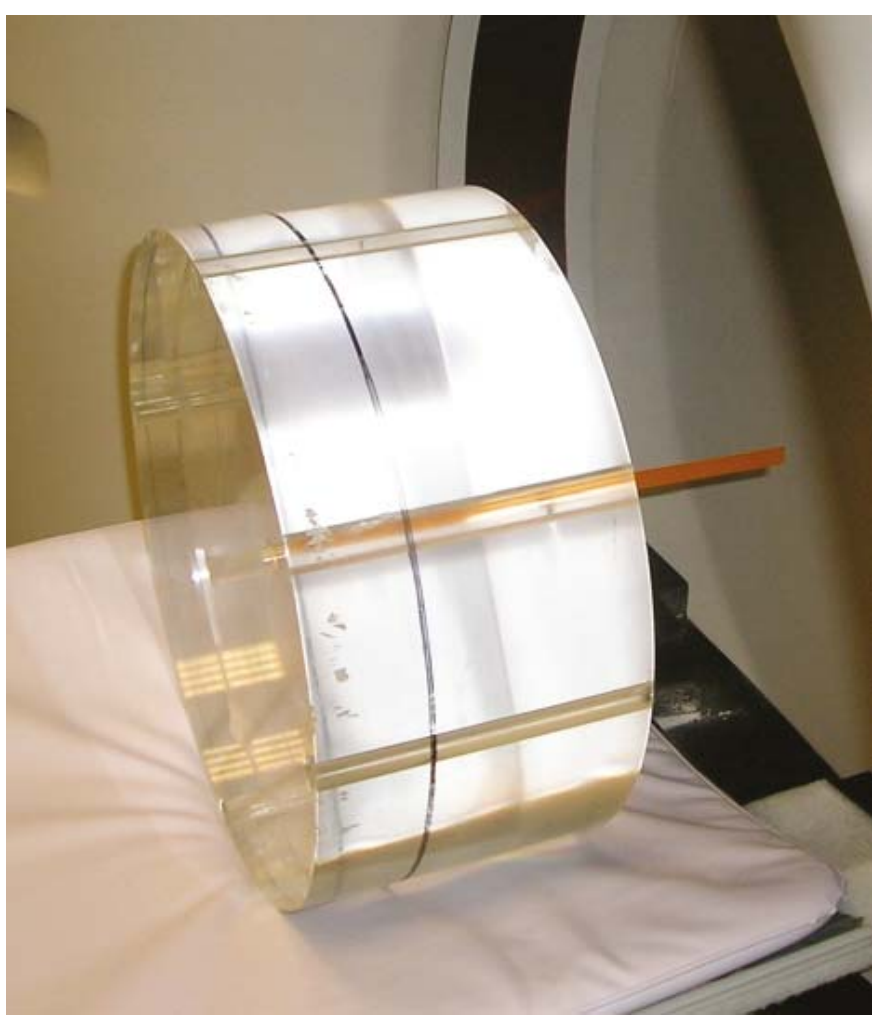

FIGURE 4. Strip of Gafchromic film inserted inside the hole of a poliymethyl methacrylate (PMMA) phantom.

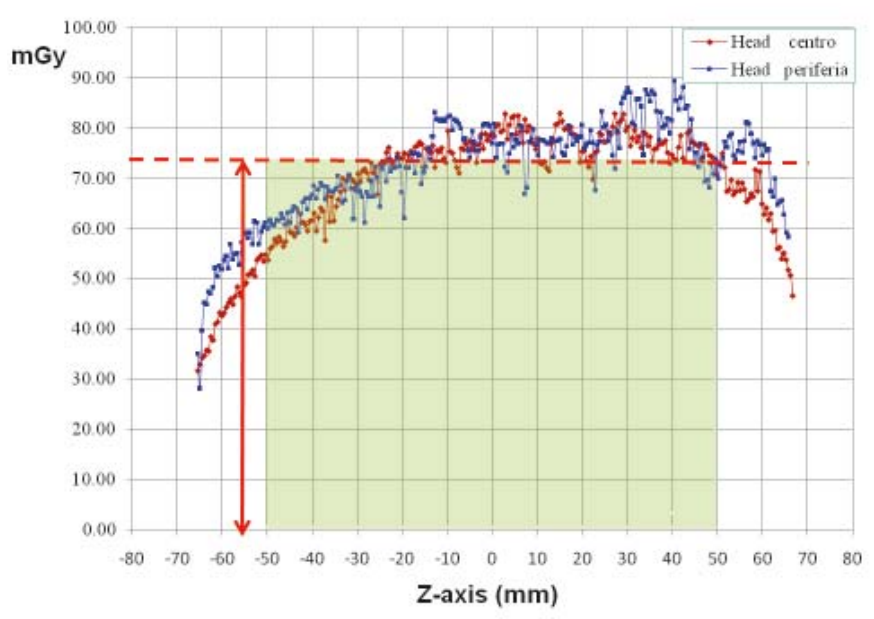

FIGURE 5. Average dose evaluation by Gafchromic film into the poliymethyl methacrylate (PMMA) phantom. of $300 \mathrm{~mm}$. Moreover, also for the measurement of CTDI in air the length of the conventional pencil chamber $(100 \mathrm{~mm})$ is inappropriate. A way to perform dosimetric measurements using conventional instrumentation is to use the ionization chamber for the measurement of the average absorbed dose and Gafchromic film for the evaluation of the actual shape of the dose profile along z-axis. In Figure 1 the shape of the dose profile is plotted; an asymmetry appears evident in the profile due to the anode heel effect. Analysing the profile FWHM was calculated to be $171.8 \mathrm{~mm}$ pointing out an overbeaming of $11.8 \mathrm{~mm}$. Z-axis geometric efficiency was also evaluated; it is defined by the International Electrotechnical Commission (IEC) ${ }^{8}$ as the ratio between the integral over the range subtended by detectors and total integral of the dose profile. The measured value of $95.4 \%$ showed for this parameter a low contribution of the penumbra effect.

In Figure 3 two different intervals of integration to evaluate the CTDI ${ }_{\text {air }}$ are presented; the value of $\mathrm{CTDI}_{\text {air,300 }}$ was $36.7 \mathrm{mGy}$ and the value of CTDI ${ }_{\text {air,100 }}$ was $34.5 \mathrm{mGy}$. The result was as expected because the longer interval takes into account also the contribution due to the tails of the dose profile.

To obtain an estimation of the absorbed dose inside the PMMA phantom, a strip of Gafchromic film was inserted in the phantom holes. The resulting value of $74.7 \mathrm{mGy}$ was compared with the value of $81.4 \mathrm{mGy}$ displayed at the CT console as $\mathrm{CTDI}_{\mathrm{vol}}$. The comparison showed a significant underestimation of the measured value probably due to the length of the PMMA phantom $(15 \mathrm{~cm})$ which is not long enough to simulate completely the effect of the scattered radiation.

\section{Discussion}

Multislice CT scanners lead to a progressive increment in the X-ray beam width along z-axis. CTDI appears to be no more suitable to represent the main dosimetric quantity in CT dosimetry. Recent publications try to find new dose parameters more representative of the technological state of art. ${ }^{9}$ 
The conventional quality assurance and the dose assessment would require an upgrade in the conventional instrumentation, but new suitable dosimeters and phantoms are not yet available for most Medical Physics Department. Nevertheless, dosimetric parameters for a CT scanner with a beam width of $16 \mathrm{~cm}$ can be estimated by means of commonly available dosimetric instrumentation.

The average absorbed dose related to a single axial rotation can be measured by conventional ionization chambers, while the geometric dose distribution along a profile in the cranio-caudal direction (Z-axis) can be evaluated exposing Gafchromic film to the primary beam.

\section{References}

1. Beslic S, Beslic N, Beslic S, Sofic A, Ibralic M, Karovic J. Diagnostic imaging of traumatic pseudoaneurysm of the thoracic aorta. Radiol Oncol 2010; 44: $158-63$.

2. Geleijns J, Salvadó Artells M, de Bruin PW, Matter R, Muramatsu Y, McNittGray MF. Computed tomography dose assessment for a $160 \mathrm{~mm}$ wide, 320 detector row, cone beam CT scanner. Phys Med Biol 2009; 54: 3141-59.

3. The measurement, reporting, and management of radiation dose in CT. Report of AAPM Task Group 23 of the Diagnostic Imaging Council CT committee. College Park: American Association of Physicists in Medicine; January, 2008

4. Comprehensive methodology for the evaluation of radiation dose in $x$-ray computed tomography. Report of AAPM Task Group 111: The Future of CT Dosimetry. College Park: American Association of Physicists in Medicine; February, 2010.

5. Rimondini A, Pozzi Mucelli R, De Denaro M, Bregant P, Dalla Palma L Evaluation of image quality and dose in renal colic: comparison of different spiral-CT. Eur Radiol 2001; 11: 1140-6.

6. Brenner DJ. Is it time to retire the CTDI for CT quality assurance and dose optimization? Medical Physics 2005; 32: 3225-6.

7. de Denaro $M$, Bregant $P$, Severgnini $M$, de Guarrini F. In vivo dosimetry for estimation of effective doses in multislice CT coronary angiography. Medical Physics 2007; 34: 3705-10.

8. IEC 60601-2-44. Particular requirements for the safety of X-ray equipment for computed tomography. In: 62B - Committee on Diagnostic Imaging Equipment, editor. Medical electrical equipment. Part 2-44. Edition 2.1. Geneva: International Electrotechnical Commission November, 2002.

9. IEC 60601-2-44. Particular requirements for the basic safety and essential performance of X-ray equipment for computed tomography. In: 62B Committee on Diagnostic Imaging Equipment, editor. Medical electrical equipment. Part 2-44. Edition 3.0. Geneva: International Electrotechnical Commission; February, 2009. 